\title{
Hard $X$ ray polarimetry with wide band Laue lens telescopes
}

\author{
Ezio Caroli, \\ INAF/IASF-Bologna \\ Via Gobetti 101, I-40129 Bologna, Italy \\ E-mail: caroliliasfbo.inaf.it
}

\section{Rui M. Curado da Silva}

LIP, Universidade de Coimbra,

P-3004-516 Coimbra, Portugal

E-mail: ruisilvalsaturno.fis.uc.pt

\section{Stefano del Sordo}

INAF/IASF-Palermo,

Via Ugo La Malfa 153, I-90146 Palermo, Italy

E-mail: Delsordo@ifc.inaf.it

John B. Stephen ${ }^{1}$,

INAF/IASF-Bologna

Via Gobetti 101, I-40129 Bologna, Italy

E-mail: stepheneiasfbo.inaf.it

\begin{abstract}
Polarimetry is today considered a key observational parameter which can be used to help solve important scientific issues that are still open in the hard $\mathrm{X}$ ray domain (above 10 $\mathrm{keV}$ ). Therefore the ability to perform high sensitivity polarisation measurements has become a mandatory requirement for the next generation of space telescopes operating in this energy range. In particular the development of new high energy focusing optics, such as wide band Laue lenses operating from $\sim 60 \mathrm{keV}$ up to several hundred $\mathrm{keV}$, with their 50-100 times better sensitivity with respect to current instrumentation, opens a real possibility to make hard X ray polarimetry an almost standard measurement. Hard X ray polarimetry can be performed using highly segmented focal plane detectors operated as scattering polarimeters. In this work we summarize results obtained by our group in a series of experiments with CZT/CdTe pixel detector prototypes operating as scattering polarimeters in the range between $\sim 100-700 \mathrm{keV}$ as well as Montecarlo evaluations of the achievable performance in polarisation measurements for Laue lens telescopes using focal planes based on CdTe/CZT pixel detectors.
\end{abstract}

The Extreme and Variable High Energy Sky,

Chia Laguna (Cagliari) , Italy

September 19-23, 2011

\footnotetext{
1 Speaker
} 


\section{Introduction}

Polarimetry in hard $\mathrm{X}$ and $\gamma$-ray energy astrophysics is still a quite unexplored domain. By measuring the polarization degree and orientation of the radiation emitted by a high energy cosmic ray source, a new, important observational parameter is obtained in addition to spectral and time variability information. Polarimetric observations in the high energy domain can provide important information on the constitution, the geometry and the physical processes responsible for the emission from various cosmic sources, e.g. Pulsars, Solar Flares, Active Galactic Nuclei, Galactic Black Holes or Gamma-Ray Bursts. In this perspective the ability to perform high sensitivity hard $\mathrm{X}$ ray polarimetry measurements has been recognized as a mandatory requirement for next space instruments operating in this range.

However, to date in the hard X- to soft $\gamma$-rays domain no dedicated polarimeters have been launched into space or flown as a balloon-borne experiment, except GAP on board IKARUS satellite and GRAPE flown on balloon in Sep. 2011 [1,2], even if some interesting results have been obtained using operating instrument $[3,4,5]$. A real challenge to make hard $\mathrm{X}$ ray polarimetry an almost standard measurements is offered by the development of new high energy focusing optics, such as wide band Laue lenses operating from $\sim 60 \mathrm{keV}$ up to several hundred $\mathrm{keV}$, with their 50-100 times expected better sensitivity with respect to current instrumentation.

\section{Scattering polarimetry with pixel detectors}

The scientific importance of polarimetry measurements shall take into account difficulties and cost in the realisation of dedicated instrument, therefore the correct strategy will be to implement this capability in conjunction with standard operation modes (spectrometry, imaging and timing) in the focal plane detector. In fact Hard $X$ ray polarimetry can be performed using highly segmented focal plane detectors optimized to operate as scattering polarimeters.

A segmented detector (i.e. a pixel detector) offers an efficient method to perform

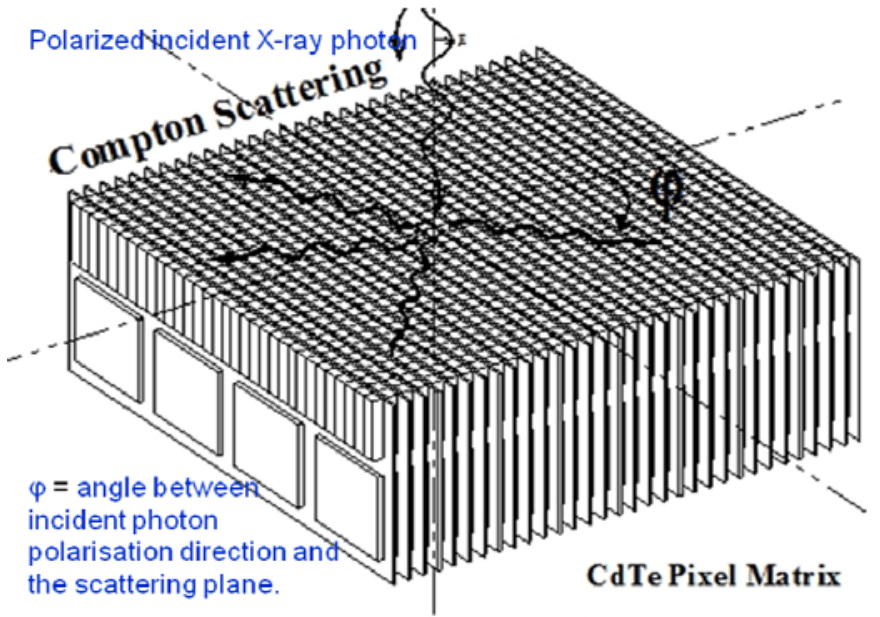

Figure 1. Compton scattering of polarized hard $X$ ray photons on a high $\mathrm{Z}$ segmented spectrometer. measurements of the linear polarisation status of impinging photons using Compton scattering because, using a proper coincidence event logic, each element/pixel can act at the same time as a scattering and as a detection unit.

Because the Klein-Nishina cross-section for linearly polarised photons exhibits an azimuth dependency for the direction of the scattered photons [6]. For polarized photon the probability distribution of 
the scattering angle will present a maximum along the direction orthogonal to the polarisation plane and a minimum in the parallel one as represented in the left plot of Figure 2.

The performance of a scattering polarimeter can be therefore evaluated by mean of its ability to measure the asymmetry of the scattering angle distribution. A well known figure of merit of a scattering polarimeter is the $\mathrm{Q}$ factor that for pixel detectors can be expressed as:

$$
Q=\frac{N_{x}-N_{y}}{N_{y}+N_{x}}
$$

where $N_{x}$ and $N_{y}$ are the counts of scattered events in two orthogonal directions.

From the spatial distribution of the scattered detected photons can be evaluated the polarisation direction and, with a proper calibration, the percentage of polarisation in the impinging photon flux.
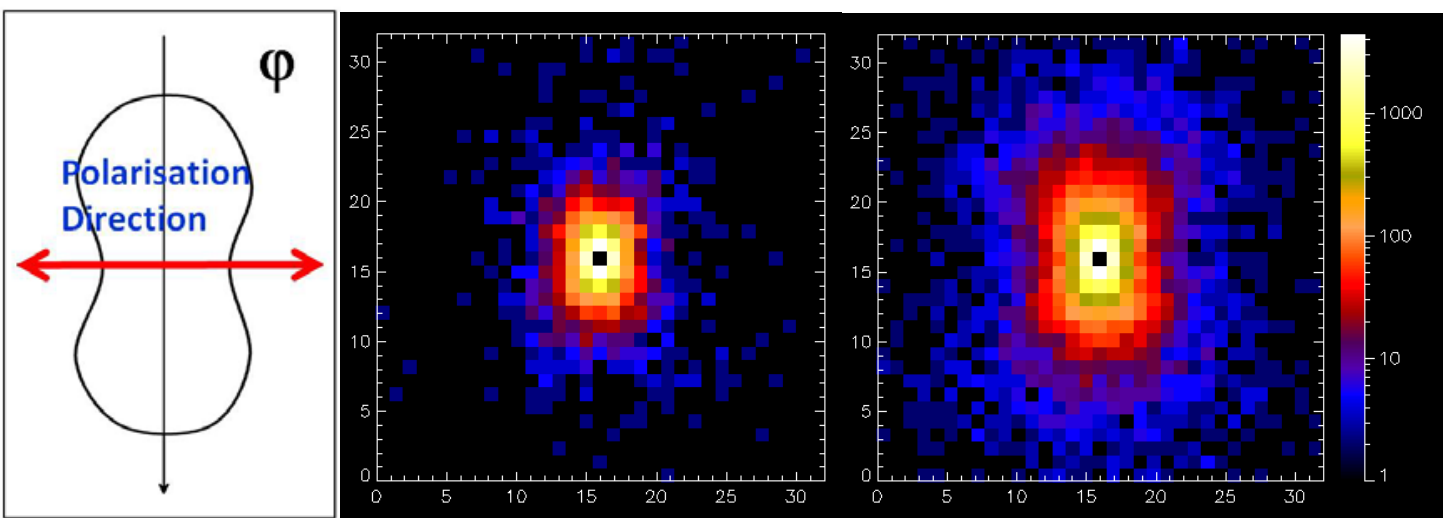

Figure 2. (left) Plot of the probability distribution of azimuth scattering angle $(\varphi)$ for polarized photons as predicted by the Klein-Nishima equation; (centre and right) the distribution of scattered events obtained by simulating monochromatic linearly $100 \%$ polarized beams impinging on a $32 \times 32$ pixels $(1 \mathrm{~cm}$ thick) $\mathrm{CZT}$ detector. The polarisation direction is horizontal, the CZT pixel size is $2 \times 2 \mathrm{~mm}^{2}$ and the beam energy is $200 \mathrm{keV}$ in the image at the centre and $400 \mathrm{keV}$ in the right one.

\section{Polarimetric tests on CZT pixel spectrometers}

Since 2001, five experiments (POLCA - POLarisation with CdTe Array - series) with a CZT pixel detector prototypes have been performed at the ESRF (Grenoble) where a high flux beam $100 \%$ linearly polarized is available at line ID15A in order to study the response as scattering polarimeter of such a detector type. The first experiment was held in 2001 using a set of few small CdTe pixel spectrometer provided by Eurorad/PHASE Laboratory (Strasbourg, France) with different thickness [7].

Due to the encouraging results, we developed a larger prototype using a $4 \times 4 \mathrm{~cm}^{2}$ sensitive area and $5 \mathrm{~mm}$ thick CZT spectrometer realized by IMARAD (now ORBOTECH, Israel) with the anode segmented in a $16 \times 16$ pixels with 2.5 pitch. This CZT pixel sensor was readout by a custom designed front end electronics based on 832 channels ASICS (eV Products) and acquired trough a multi-parametric electronics with coincidence logic able to handle up 128 channels (see Figure 3). Using this detection system three more experiments have 

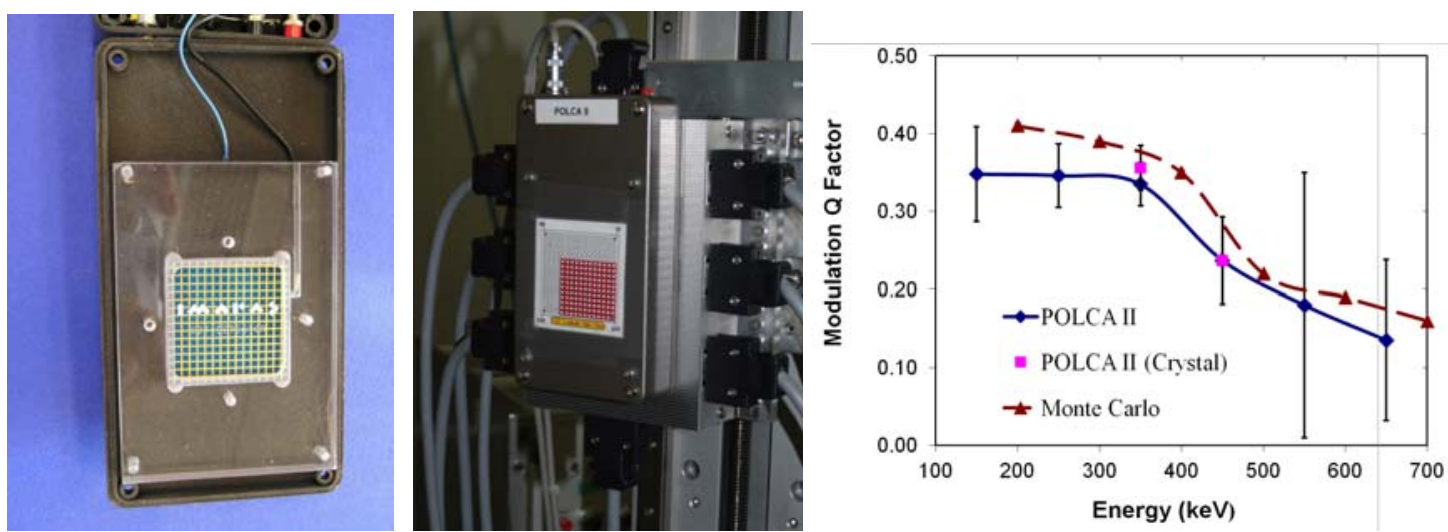

Figure 3. (left) The IMARAD CZT pixel sensor from the top surface that surface (i.e. the cathode); (centre) The CZT detector box connected to the analogue front end metallic box in measurement configuration inside the ESRF ID 15A line experimental hutch; (right) summary of the measured Q factor in the POLCA II experiment as function of energy compared with MonteCarlo results.

been performed at the ESRF facility devoted to study different aspects and parameters affecting the performance as scattering polarimeter of a CZT pixel spectrometer (Figure 3) [8].

In particular with the $3^{\text {rd }}$ ESRF experiment (LaPOLCA - Laue lens POLCA) we have evaluated the polarimetric performance of our prototype coupled with Laue lens using a mosaic of single $\mathrm{Cu}$ crystal to simulate the response of rings with different radius of the lens focussing the polarized beam on the detector. This test was conceived to evaluate possible systematic effects of the Laue diffraction on the polarisation status of the impinging photon. In fact the
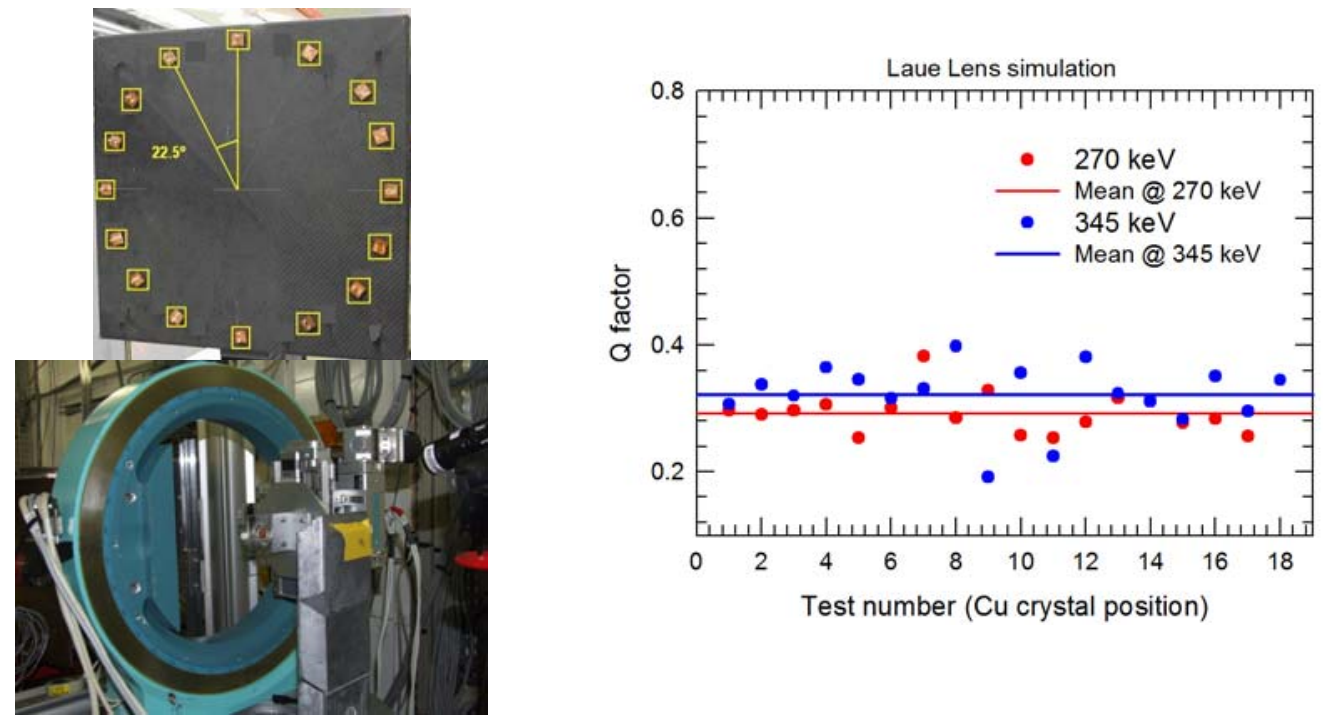

Figure 4. (left) The Laue lens simulator in the experiment hutch of the ESRF ID15 B beam line (left bottom) and the scheme of the simulated 18 Laue ring positions (left top). (right) The modulation factor Q obtained for each $\mathrm{Cu}$ position in the Laue lens simulation at two energies that are compatible with a constant value within a percent.

results have demonstrated that in the hard $\mathrm{X}$ ray regime the Laue diffraction does not affect (within a percent) the measured polarisation (Figure 4) [9].

The last ESRF experiment with the POLCA (POLCA III) detection system was manly dedicated to assess its polarimetric performance at different energies as a function of the angle between the detector surface axis and the beam polarisation direction and to evaluate its sensitivity to the beam polarisation level (Figure 5) [10]. 
Since 2010 a collaboration with CEA/Saclay (France) is born to perform further experiments on hard $\mathrm{X}$ ray polarimetry with CdTe pixel spectrometer using a new detector module (the Caliste module, [11]) with sub millimetre spatial resolution, high spectroscopic performance and response uniformity developed by this group in the framework of the Simbol $\mathrm{X}$ satellite proposal. In May 2011 we had a first test campaign at ESRF (ID15A) to evaluate the polarimetric performance of this innovative type of pixel detector. In particular these measurements will allow us to evaluate the improvement that we can achieve in polarimetric performance (in term of sensitivity and efficiency) using thin detector layers coupled with high energy resolution and fine pixel scale, giving to us important information to optimize the design of a next generation of focal plane detectors. The first really great results of this experiment has been presented at the 2011 IEEE NSS/RTSD conference in Valencia (Spain) [12].
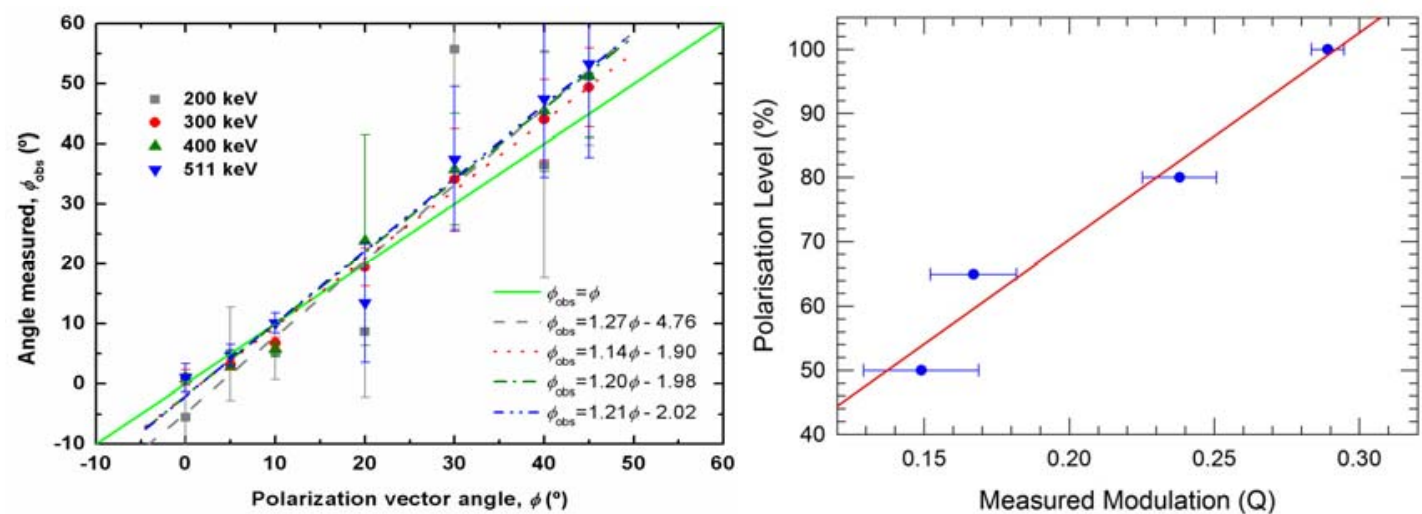

Figure 5. (left) The measured polarization vector angle $\left(\varphi_{\mathrm{obs}}\right)$ as a function of the effective beam polarization angle $\left(\varphi_{\text {beam }}\right)$. (right) Sensitivity evaluation of the CZT pixel polarimeter: the $\mathrm{Q}$ factor obtained by varying the polarization level of a $400 \mathrm{keV}$ beam from $100 \%$ to $50 \%$.

\section{Laue telescope polarimetry performance}

As an example we present some evaluation on achievable performance in high energy polarimetry for a balloon Hard X-ray focusing Telescope (HAXTEL-B) based on wide bandpass Laue optics [13] with a focal plane based on a thick $(10 \mathrm{~mm})$ pixel CZT detector. The results are based on MonteCarlo simulation between 50-350 keV and on experimental results obtained with the pixel CZT detector prototype used in the PolCA series experiments.

Another very important figure of merit for a scattering polarimenter is the so called Minimum Detectable Polarisation (MDP). MDP indicates when one may be confident that the signature of polarization has been detected: i.e., that the source is not un-polarized and for a polarimeter to be effective it should be significantly smaller than the degree of polarization to be measured. MDP at 99\% confidence level can be expressed by [14]:

$$
M D P_{99 \%}=\frac{4.29}{A \cdot \varepsilon \cdot S_{F} \cdot Q_{100}} \sqrt{\frac{A \cdot \varepsilon \cdot S_{F}+B}{T}}
$$

where $\mathrm{Q}_{100}$ the modulation factor for a $100 \%$ polarised source, $\varepsilon$ the detection efficiency for scattered events, A the polarimeter detection area in $\mathrm{cm}^{2}, \mathrm{~S}_{\mathrm{f}}$ the source flux (photons $/ \mathrm{s} / \mathrm{cm}^{2}$ ), B is the background flux (counts/s) and $\mathrm{T}$ the observation time in seconds. 


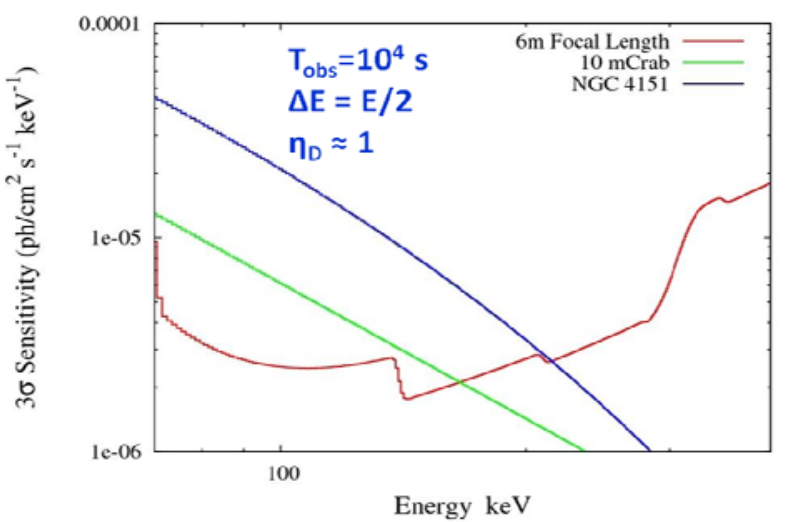

Figure 6. The expected continuum sensitivity (red curve) of the HAXTEL-B telescope for $\sim 3$ hours observation time compared with the cosmic ray fluxes from typical high energy sources. The detector efficient $\left(\eta_{D}\right)$ is assumed $100 \%$.
As a case study we refer to the HAXTEL-B telescope proposed as a balloon borne payload [15]. In the baseline configuration, the HAXTEL-B had as a focal length of $6 \mathrm{~m}$, and the Laue optics is made by a set of $\mathrm{Cu}(111)$ crystals with $3^{\prime}$ mosaic spread. The proposed Laue lens allow to focus hard $\mathrm{X}$ rays in the $70-350$ $\mathrm{keV}$ range.

The expected sensitivity of the HAXTEL-B telescope obtained with Monte Carlo simulations for typical balloon observation time scale is reported

in Figure 6. In this evaluation the used background was derived by a scaling from the SAX/PDS measured values.

Using the equation (2) it is possible to provide an evaluation of the minimum detectable polarization achievable in $10000 \mathrm{~s}$ of the HAXTEL-B payload for different configuration of the Laue lens and the focal plane detector. To perform this exercise we have assumed as focal plane a mosaic of CZT pixel spectrometer of $5 \mathrm{~mm}$ thickness. The first row of Table 1 report the MPD estimated for the baseline HAXTEL-B design in which the lens is made of $\mathrm{Cu}$ mosaic crystals and the focal plane is segmented in $2 \times 2 \mathrm{~mm}^{2}$ pixels.

Table 1. HAXTEL-B MDP evaluation for $100 \mathrm{mCrab}$ source in $10000 \mathrm{~s}$

\begin{tabular}{l|c|c|c|c|c}
\hline Laue Lens type & CZT pixel size & $\begin{array}{c}\text { CZT vertical } \\
\text { segmentation }\end{array}$ & $100-200 \mathrm{keV}$ & $150-250 \mathrm{keV}$ & $250-350 \mathrm{keV}$ \\
\hline \hline Mosaic crystals & $2 \times 2 \mathrm{~mm}^{2}$ & $5 \mathrm{~mm}$ & $13 \%$ & $11 \%$ & $9 \%$ \\
\hline Curved crystals & $2 \times 2 \mathrm{~mm}^{2}$ & $5 \mathrm{~mm}$ & $6 \%$ & $5 \%$ & $4 \%$ \\
\hline Curved crystals & $0.5 \times 0.5 \mathrm{~mm}^{2}$ & $0.5 \mathrm{~mm}$ & $3 \%$ & $2.5 \%$ & $2 \%$ \\
\hline
\end{tabular}

The average effective area of the HAXTEL telescope used in the evaluation range from $100 \mathrm{~cm}^{2}(100-200 \mathrm{keV})$ down to $\sim 30 \mathrm{~cm}^{2}$ for $250-350 \mathrm{keV}$. The double event efficiency strongly increase with the detector pixel/voxel scale: in the baseline $\left(2 \times 2 \mathrm{~mm}^{2}\right.$ pixel, no vertical segmentation), simulations indicate values from $12 \%$ at $200 \mathrm{keV}$ up to $17 \%$ at $350 \mathrm{keV}$. The MDP values in the last two rows of the table are obtained scaling the simulated (first row) ones considering the HAXTEL-B performance improvement achievable with the implementation of curved crystals [16] to build the Laue lens (50-100 times smaller PSF) and a finer segmentation of the focal plane. The use of curved crystals will allow to drastically decrease (up to a factor of 100) the background because of the smaller PSF, while a focal plane detector finer segmentation achievable with new $3 \mathrm{D}$ configurations would increase both the modulation factor $\mathrm{Q}(\times 1.5)$ and the scattered event detection efficiency $\varepsilon(\times 1.5-2)$. 


\section{Conclusions}

CZT detectors used as wide band Laue lens focal planes are potentially well suited to perform very sensitive polarimetric measurements in the hard $\mathrm{X}$ and soft gamma ray domain. Both simulated and experimental data support this conclusion. Experimental measurements performed with mosaic $\mathrm{Cu}$ crystals in the Laue configuration show that polarization is not affected by Laue diffraction. Furthermore experimental data confirm that very good modulation $\mathrm{Q}$ factor values (up to 0.5 ) can be obtained in the hard X ray domain with 3D detectors with fine segmentation allowing low MDP to be achieved even for a balloon borne Laue lens telescope.

\section{References}

[1] D. Yonetoku, et al., The Astrophysical Journal Letters, Vol. 743, L30, 2011

[2] T.P. Connor, et al., Proc. of SPIE, Vol. 7732, p. 77324E-1, 2010

[3] A. J. Dean, et al, Science, Vol. 321, No 5893, p. 1183, 2008.

[4] M. Forot, et al., The Astrophysical Journal., Vol. 688, p. L29, 2008.

[5] P. Laurent et, al. , Science, Vol. 332, p. 438, 2011

[6] F. Lei, A. J. Dean, and G. L. Hills, Space Science Review., Vol. 82, p. 309, 1997.

[7] R. M. Curado da Silva, et al., IEEE Trans. on Nucl. Sci., Vol. 51, No 5, p. 2478, (2004).

[8] R.M. Curado da Silva, et al, J. Appl. Phys. Vol. 104, p. 84903, doi: 10.1063/1.3000092, (2008).

[9] E. Caroli, et al., IEEE Trans. on Nucl. Sci., Vol. 56, No 4, p. 1848, (2009).

[10]R.M. Curado da Silva, et al., IEEE Trans. on Nucl. Sci., Vol. 58, No 4, p. 2118, (2011).

[11] O. Limousin, et al., Nucl. Instr. and Meth. in Phys. Res. A, Vol. 647, p. 46, (2011)

[12] O. Limousin, et al., "Polarisation performance of the CdTe/CZT Caliste detector modules", Poster RTSD.S 316 presented at IEEE NS MIC and RTSD Conference, 23-29 October 2011, Valencia, Spain, in publication on the proceeding records.

[13] F. Frontera, et al., Experimental Astronomy, Vol. 20, p. 241, 2005.

[14] M. C. Weisskopf, et al., in "Neutron Stars and Pulsars", Astrophysics Space Science Library, Vol. 357, p. 589, 2009.

[15] F. Frontera, et al., Proc. of ' $19^{\text {th }}$ ESA Symposium on European Rocket and Balloon Programmes and Related Research", Bad Reichenhall, Germany, 7-11 June 2009, ESA SP-671, (2009)

[16] R. Camattari, et al., Proc. SPIE, Vol. 8147, 81471G ; doi:10.1117/12.895311 (2011) 